\title{
Effectiveness and feasibility of home-based telerehabilitation for community-dwelling elderly people in Southeast Asian countries and regions: a systematic review
}

\author{
Takashi Saito $^{1}$ (D) Kazuhiro P. Izawa ${ }^{2} \mathbb{D}$
}

Received: 3 February 2021 / Accepted: 17 February 2021 / Published online: 25 March 2021

(c) The Author(s), under exclusive licence to Springer Nature Switzerland AG 2021

\begin{abstract}
This systematic review aimed to evaluate the impact of home-based telerehabilitation on physical function among communitydwelling elderly people in Southeast Asian countries and regions, and to investigate its feasibility. A systematic electronic literature search was conducted in PubMed/MEDLINE and PEDro according to PRISMA guidelines. Randomized controlled trials conducted in the area that involved elderly people and any physical function indexes were included. Home-based telerehabilitation was defined as a specific remote rehabilitation intervention that used any kind of technological device allowing healthcare professional/patient interaction. Information regarding the effect and feasibility (intervention completion rate) of home-based telerehabilitation was extracted from eligible articles. We used the Revised Cochrane risk-of-bias tool for randomized trials to assess methodological quality of the included articles. Eventually, six studies were included as eligible articles. The overall risk of bias judgement was assessed as "High" in five studies. All studies were conducted in either China or South Korea, and heterogeneity in terms of participants' health condition and intervention regimen was observed across the studies. Our narrative-based analysis showed that compared with conventional rehabilitation, either equal or better effects on physical function were reported across the six studies. The intervention completion rates were $81 \% \pm 11$ on average (range 59-96\%). Although we could not obtain conclusive evidence due to limited relevant information with heterogeneity across the studies, our findings suggest that home-based telerehabilitation can be a strategy for rehabilitation service delivery with acceptable feasibility comparable to conventional rehabilitation for elderly people in the area.
\end{abstract}

Keywords Aged $\cdot$ Southeastern Asia $\cdot$ Internet-based intervention $\cdot$ Physical functional performance $\cdot$ Telerehabilitation

\section{Introduction}

Southeast Asian countries and regions are facing the challenge of global aging. In 2019, the number of elderly people in the area aged 65 years or older was estimated at approximately 261 million [1] This figure is expected to rapidly grow to more than 573 million by 2050 , the largest increase ever in the world [1]. With the accompanying rise in multimorbidity and age-related health issues such as frailty, population aging will lead to a higher absolute number of

Takashi Saito

takashi-riha@live.jp

1 BIKOU Visiting Nursing and Rehabilitation Station, 1-11-13, Tokiwadai, Itabashi-ku, Tokyo 173-0004, Japan

2 Department of Public Health, Graduate School of Health Sciences, Kobe University, Kobe, Japan elderly people with lower physical function, disability and poor quality of life [2]. Consequently, these elderly people susceptible to frailty impose a burden on health care systems and are becoming a threat to their own sustainability [2-4]. Effective, feasible and resource-sensitive strategies are needed to tackle this health issue in the area.

It has been well documented that hospital- and institution-based rehabilitation services, which typically include physical exercise, can be effective both from a health perspective (e.g., mitigating functional decline) and economic perspective (e.g., reducing health care costs) for elderly people $[5,6]$. The unfortunate reality, however, is that numerous factors such as a shortage of rehabilitation personnel [7] and logistical (e.g., distance to service) and economic (e.g., affordability of services) barriers [8] prevent community-dwelling elderly people from receiving rehabilitation services in many Asian countries, especially in low- and middle-income countries and resource-restricted 
areas. To address this gap, new strategies to deliver rehabilitation services are necessary.

One promising solution to address this gap may be a telehealth care system, which uses information and communication technologies (ICTs) such as smartphones, videophones and the internet to provide cost-effective, quality and flexible health care services across geographic, time and economic barriers $[9,10]$. Home-based telerehabilitation (HBT), one of the emerging fields of telehealth, is a strategy of rehabilitation service delivery that can be defined as the use of ICTs by a health professional to provide evaluation and rehabilitation interventions for people living at home [11, 12]. This new strategy has been promoted as a solution to the challenge created by an aging population [13, 14]. Additionally, this strategy, which can remotely provide rehabilitation services without or lowered physical contact with elderly people, could be an alternative to conventional, face-to-face and hands-on rehabilitation services during the coronavirus disease 2019 pandemic in which physical distancing is necessary to prevent infection of both vulnerable elderly people and health service providers with the virus [15].

Given the novelty of this service delivery strategy, careful consideration is required prior to implement the interventions. The Canadian Physiotherapy Association provides a 10-item checklist and recommends that practitioners ensure the validity of launching the services [16]. The first question in the checklist asks, "Is tele-rehabilitation appropriate for this client?" The appropriateness of adapting HBT intervention should be judged by health professionals taking into account broad aspects such as effectiveness, feasibility, client preference and ethical and privacy issues [17]. Having a broad range of expertise and evidence that allows health professionals to make sound judgements on the appropriateness of implementing an HBT intervention is crucial.

Over the past decades, several systematic reviews have reported supporting evidence on the effects and feasibility of HBT intervention [10,18-24]. This literature provides an important basis for judging the validity of implementing the interventions. Nevertheless, from the context of global aging in Southeast Asian countries and regions, there are some difficulties in using and adapting this evidence. First, the scopes of these previous systematic reviews [18-22] were diseasespecific examinations and validation of HBT among varied age groups; thus, these findings may not be generalizable to specific older age generations. Second, although a few systematic reviews $[10,23,24]$ focused on elderly populations, they were mainly based on randomized controlled trials (RCTs) conducted in Western countries; thus, it is unclear whether these findings can be applicable to Asian countries and regions with different internet use $[25,26]$ and different cultures and languages than Western countries.

Therefore, important issues not yet addressed in previous studies are the effectiveness and feasibility of HBT among elderly people in Southeast Asian countries and regions. Thus, the aims of this systematic review were twofold. First, we evaluated the impact of HBT intervention on physical function among community-dwelling elderly people in Southeast Asian countries and regions. Second, to discuss the feasibility of HBT and potential contributing factors, we investigated and summarized completion rates and reasons for withdrawal from the HBT interventions along with safety-related data.

\section{Method}

We conducted and reported this systematic review in accordance with the Preferred Reporting Items for Systematic Reviews and Meta-Analyses (PRISMA) guidelines [27]. See Online Resource 1 for a checklist of each component.

\section{Search strategy}

An electronic literature search was conducted in PubMed/ MEDLINE and PEDro on 23 September 2020. The literature search was limited to articles written in English or Japanese and published from 1 January 2000 to the date of the search. We used a search strategy that combined Mesh (Medical Subject Heading) and free keywords and connected them with Boolean conjunctions (OR/AND). Keywords included wording such as telerehabilitation, "internet-based intervention", "far east" and the name of each Southeast Asian country and region. The search strategy and keywords were elaborated through discussion and peer review between the two authors. Details of the search terms and search strategies in PubMed and PEDro are shown in Online Resource 2.

\section{Eligibility criteria}

In this systematic review, we only included RCTs that studied impacts of HBT intervention on physical function among elderly people in Southeast Asian countries and regions. Specific eligibility criteria for inclusion were defined according to the PICO framework (population, intervention, comparator and outcome) (Table 1).

\section{Study selection}

Identification of potentially relevant papers and eligibility assessment were performed independently in a standardized blinded manner by the two authors. This process was divided into two phases. First, the titles, abstracts and authors' affiliations were screened along with eligibility criteria by the authors. They assessed the relevance of each article for inclusion according to three options: "definitely relevant", "possibly relevant" and "not relevant". Second, 
Table 1 Eligibility criteria for inclusion

\begin{tabular}{|c|c|}
\hline dy setting & $\begin{array}{l}\text { The location of the study setting was required to be in Southeast Asian countries and regions that consist of China, Hong Kong, } \\
\text { Macau, Tibet, Korea, Mongolia, Taiwan, Japan, Borneo, Brunei, Cambodia, Indonesia, Laos, Malaysia, Myanmar, Philippines, } \\
\text { Singapore, Thailand, Timor-Leste and Vietnam }\end{array}$ \\
\hline Participants & $\begin{array}{l}\text { The participants were community-dwelling elderly people living at home in Southeast Asian countries and regions. Average age } \\
\text { of the participants was } 60 \text { years and older. No restriction was imposed on the participants' health conditions }\end{array}$ \\
\hline Interventions & $\begin{array}{l}\text { Definition of telerehabilitation was quoted from a previous study }{ }^{12} \text { describing the interventions as: } \\
\text { provided by means of any kind of technological device allowing healthcare professional/patient interaction both on-line or off- } \\
\text { line; } \\
\text { provided by healthcare professionals or caregivers through remote supervision; } \\
\text { including at least one specific intervention targeted to rehabilitation (e.g., teletraining at home, teleconsultation, teletreatment, } \\
\text { and telemonitoring or telesurveillance) }\end{array}$ \\
\hline Comparator & $\begin{array}{l}\text { Comparators included either usual rehabilitation interventions, which were provided in-person in hospital/institution setting, } \\
\text { educational intervention, or no specific interventions }\end{array}$ \\
\hline Outcomes & $\begin{array}{l}\text { Any kinds of physical function/motor performance (e.g., mobility, balance, strength, walking) were required to be reported in the } \\
\text { articles }\end{array}$ \\
\hline
\end{tabular}

they screened the full text of the articles judged as "definitely relevant" or "possibly relevant" in the 1st phase and made a final judgement on the articles according to two options: "relevant" or "not relevant". In both phases, if disagreements regarding judgement of relevance arose, the two authors reached a final decision through negotiation. To assess the degree of agreement between authors, we calculated Kappa statistics for both phases. In our calculations, "definitely relevant" and "possibly relevant" in the 1 st phase were merged into "definitely or possible relevant". A $P$ value of $<0.05$ was considered to indicate statistical significance.

\section{Data extraction}

Data were extracted by TS and checked by KPI using a unified form. The extracted information included author, publication year, study setting (country or region), participants' data (age, sex and medical condition), interventions data (ICT devices and platform, regimens of the interventions, presence or absence of in-person interventions during HBT, compared intervention and effects) and feasibility data (intervention completion rate, reasons for withdrawal from the intervention and adverse events during the intervention). In terms of potential factors contributing to feasibility, safety-related issues, which can be roughly divided into medical issues and technological issues (problems with ICTs) [17], are reported as the potential factors influencing compliance with the exercise intervention [17, 28]. Thus, we extracted these data along with medical safety measures used during the interventions and the participants' skills and experience with ICTs.

\section{Quality assessment}

The two authors independently evaluated the methodological quality of the included articles using the Revised Cochrane risk-of-bias tool for randomized trials (ROB2) [29]. The ROB2 tool provides a framework for considering the risk of bias in the findings of any type of randomized trial. Five domains of bias are considered in ROB2: (1) bias arising from the randomization process, (2) bias due to deviations from intended interventions, (3) bias due to missing outcome data, (4) bias in measurement of the outcome and (5) bias in selection of the reported result. Each domain consist of several signaling questions for assigning one of three levels of risk of bias judgement to each domains: Low risk of bias, Some concerns or High risk of bias. These judgements of each domain eventually enable the assessor to make overall risk of bias judgements on each trial.

\section{Results}

\section{Study selection}

The study selection process is illustrated in the Fig. 1. In total, 422 articles without duplication were identified through the literature search. Of these 422 articles, 30 were assessed as "definitely relevant" or "possibly relevant", and the remaining articles were considered "not relevant" in the 1st phase. After screening the 30 full-text articles in the 2nd phase, 6 studies [30-35] were identified as eligible studies for inclusion in this systematic review. Kappa statistics for judgements in the 1st and 2 nd phase were $0.629(P<0.001)$ and $0.286(P=0.025)$, respectively. All disagreements on judgements were reconciled through negotiation between the authors.

\section{Overview of included studied}

\section{Characteristics of studies}

Characteristics of the six eligible RCTs are summarized in Table 2. All studies were conducted in either China [32-34] 


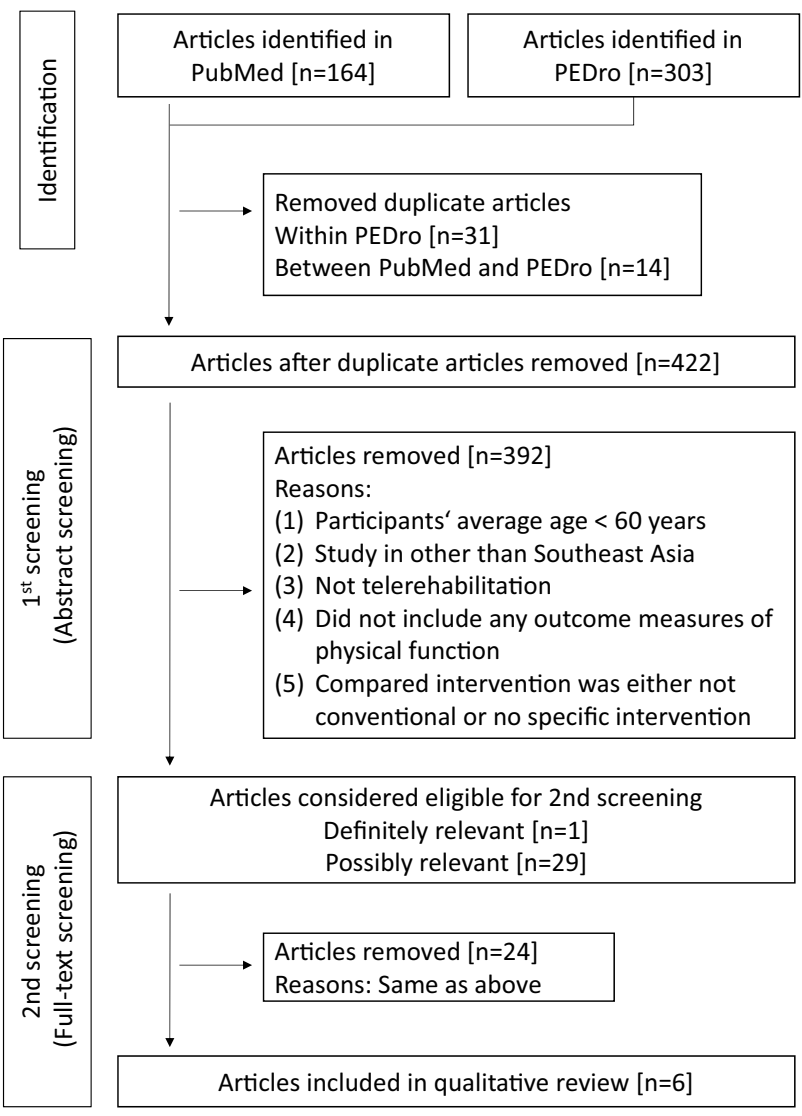

Fig. 1 Flow diagram of the study selection process in the systematic review. An electronic literature search was conducted in PubMed/ MEDLINE and PEDro on 23 September 2020. In total, 422 articles without duplication were identified through the literature search. Of these articles, 30 were assessed as "definitely relevant" or "possibly relevant", and the remaining articles were considered "not relevant" in the 1st phase. After screening of the 30 full-text articles in the 2nd phase, 6 studies were identified as eligible studies for inclusion in this systematic review

or South Korea [30, 31, 35]. Participants' health conditions, sample size and sex ratio varied widely across the studies. The health conditions included heart diseases [32, 33], pulmonary disease [31], cancer [35], stroke [34] and community-dwelling elderly without specific conditions [30], and the sample sizes and ratio of females (\%) ranged from 13 to 50 participants and $0-55 \%$, respectively. The ICT devices used in the HBT interventions were smartphones [31-33, $35]$ and personal computers [30, 34]. Four of the six studies [31,33-35] developed a specific platform to administer their HBT intervention, and the other two studies [30, 32] used the common free apps Skype [30], QQ and WeChat [32].

The main component of the HBT regimens also varied across the studies: resistance exercise [30], aerobic exercises $[31,33]$, combination of aerobic and resistance exercises $[32,35]$ and physical exercise and electromyography-triggered neuromuscular stimulation technique [34] (Table 2).
Of the 6 studies, $3[30,31,33]$ were accompanied by faceto-face interventions during the HBT interventions. The compared interventions ranged from education and regular follow-up [30, 32, 33], outpatient rehabilitation services [34] and self-monitoring approach to physical activity using a conventional pedometer [35] to no specific intervention [31]. Given the heterogeneity in the study characteristics, it was not appropriate to carry out a meta-analysis. Thus, we conducted a narrative analysis of the six eligible studies.

\section{Assessment of the risk of bias}

Figure 2 summarizes the results of the risk of bias assessment using ROB2. The overall risk of bias judgement was assessed as "High" in all but one study [34], which was assessed as having "some concerns". In terms of the results of risk of bias due to deviations from the intended interventions (domain 2), none of the 6 studies could blind the participants because they were always involved in and aware of the HBT. Thus, the risk of bias in domain 2 was assessed as "High" in most studies [30-33, 35], with one exception [34], which (1) provided identical interventions with only a difference in service delivery strategy between groups and (2) applied intent-to-treat analysis to minimize the influence of the bias. The risk of bias due to missing outcome data (Domain 3) was judged as "High" in 5 studies [30-33, 35], due to their considerably high attrition rates. In terms of the risk of bias in measurement of the outcome (Domain 4), a certain discrepancy in assessor blinding was found across the studies: assessor blinding was confirmed in three studies [31, 32, 34], but no specific description of its presence or absence was found in the other three studies [30, 33, 35]. All studies, however, were judged as having "Low" risk in domain 4 because their outcome measure was not participant reported, which can be potentially influenced by knowledge of the intervention received, leading to a judgement of at least "Some concerns" [29]. Regarding the risk of bias in selection of the reported result (Domain 5), only one study [34] had a protocol paper [36] published in advance. There was, unfortunately, a discrepancy between them in reported outcomes $[34,36]$. Thus, risk of bias in domain 5 was judged as "High" or "Some concerns" in all six studies.

\section{Effects of home-based telerehabilitation}

The most common outcome used in the trials was the 6-min Walk Test (6MWT) [31-33], followed by a combined physical performance test $[30,35]$ and Berg Balance Scale (BBS) [35] (Table 2). Overall, either equal or better effects on each outcome were reported across the six studies in comparison with conventional rehabilitation. In the study of Peng et al. [32], for example, an 8-week HBT intervention of aerobic and resistance exercise for elderly people with heart failure 


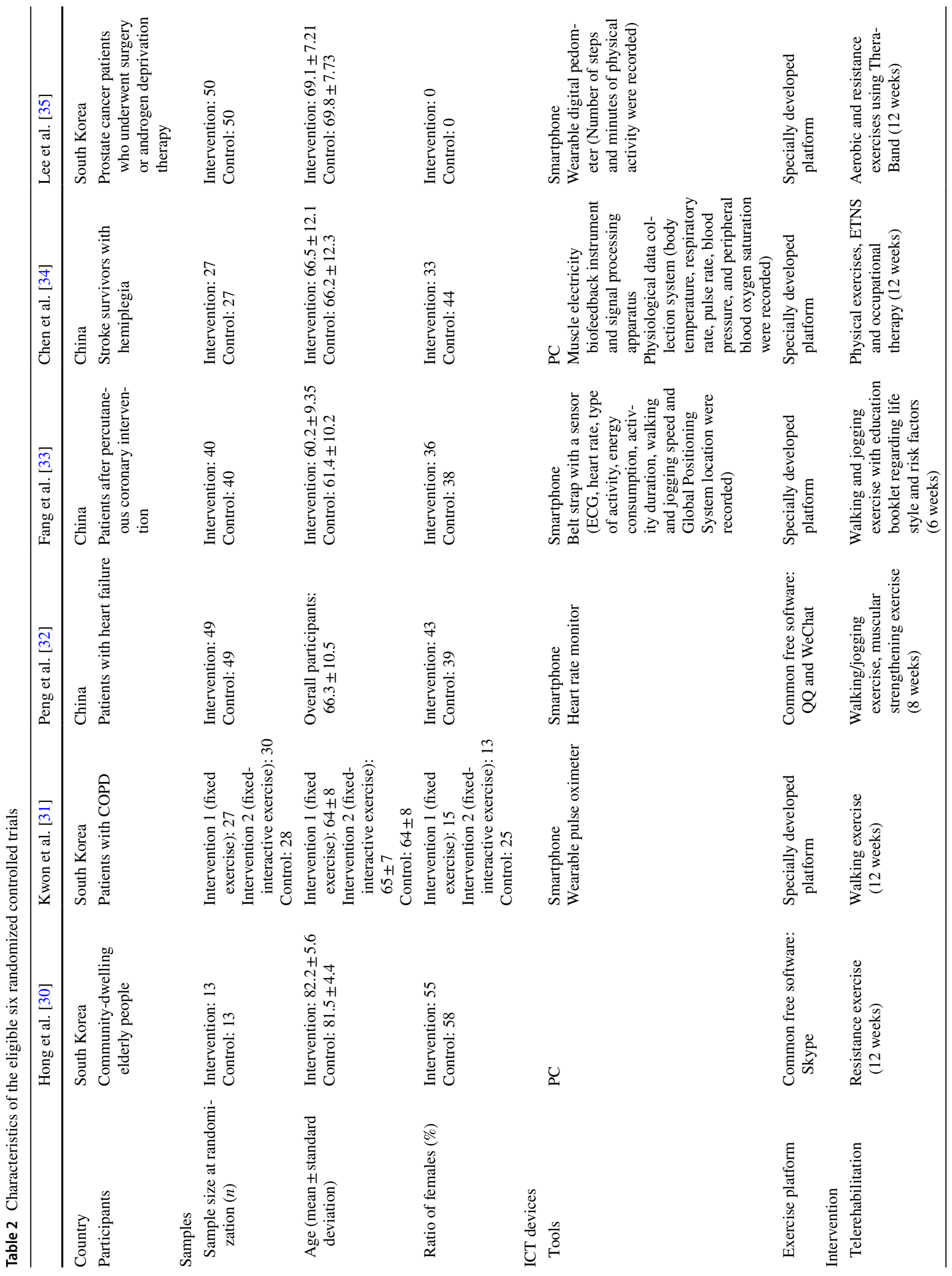




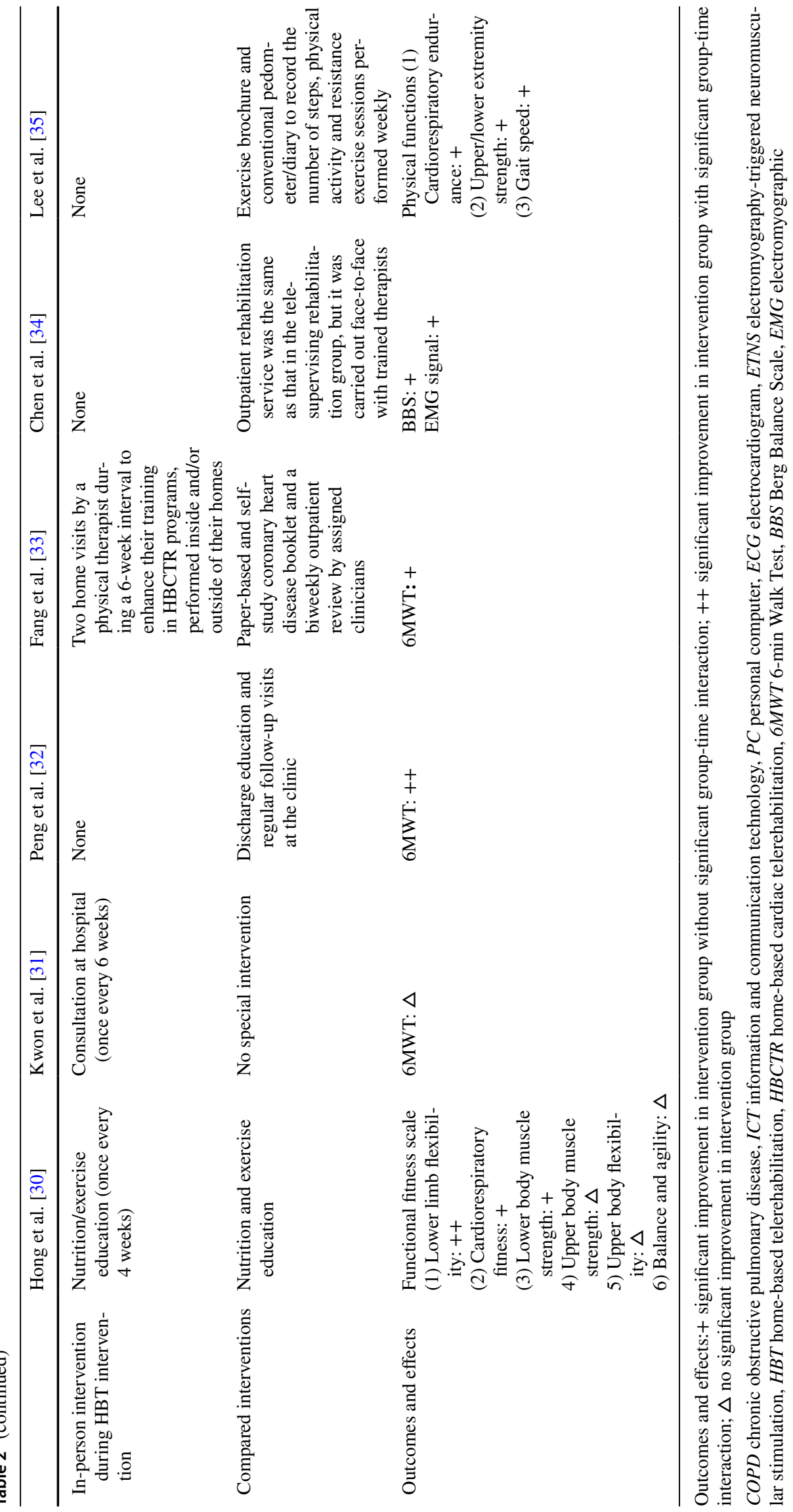




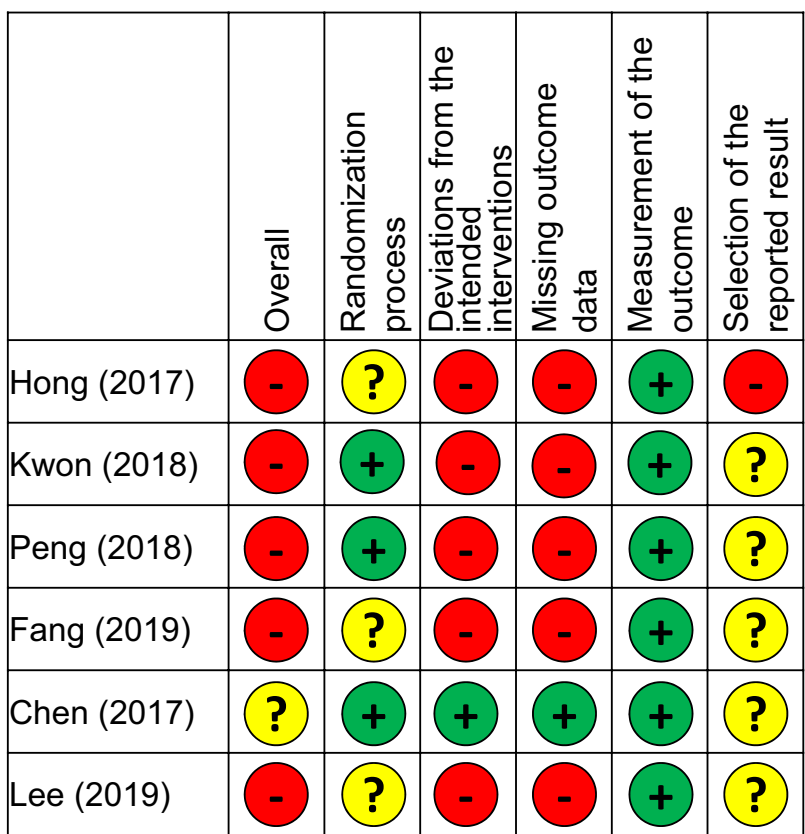

Low risk

Some concerns

High risk

Fig. 2 Summary of the results of the risk of bias assessment using the Revised Cochrane risk-of-bias tool for randomized trials

was conducted, and its effects on the 6MWT were evaluated. The results showed that the $6 \mathrm{MWT}$ in the intervention group improved significantly after 8 weeks of intervention (baseline: $407 \mathrm{~m}$, post intervention: $419 \mathrm{~m}$ ), and its effects persisted for 16 weeks after the interventions $(418 \mathrm{~m})$ with a statistically significant group-time interaction. In contrast, the study of Kwon et al. [31] was the sole study in this systematic review to report a negative outcome of HBT interventions. They evaluated the effects of HBT intervention, which prescribed aerobic exercise using smartphone and a specially developed app (efil breath) for elderly people with chronic obstructive pulmonary disease. As a result, they were not able to find significant improvement in their outcome measure, the 6MWT, after a 12-week HBT aerobic intervention.

\section{Feasibility of home-based telerehabilitation}

\section{Completion rate, reasons for withdrawal and adverse events (Table 3 )}

Completion rates were reported in all studies: an average of $81 \% \pm 11$ (range 59-96\%) in the intervention group and $86 \% \pm 4.1$ (range 79-92\%) in the control group (Table 3). Most studies [30-34] described the reasons for withdrawal, such as no-shows, withdrawal of informed consent and loss to follow-up. Specific reasons or background information for these withdrawals, however, was not described clearly in all studies. Two studies [32, 34] clearly reported that no adverse events occurred during the HBT interventions, whereas the other 4 studies [30, 31, 33, 35] did not specifically describe adverse events.

\section{Potential contributing factors to feasibility (Table 4)}

We summarize the data on potential contributing factors to feasibility, specifically medical-related safety issues (safety measures during the HBT intervention) and technologyrelated safety issues (participants' ICTs skills/experience) in Table 4. As a safety measure, in the four studies [31-34] in which participants with cardiovascular and pulmonary diseases were recruited, a physiological data sensor was used during exercise that commonly collected heart rate and $\mathrm{SpO}_{2}$ data. Additionally, the protocol of two studies [32, 34] also required caregivers to accompany their participants during the intervention. Finally, we found that only four studies [31-33, 35] imposed some kind of inclusion criteria based on the participants' ICTs skills/experience. No restriction regarding this prerequisite condition was imposed in the other two studies [30, 34].

\section{Discussion}

\section{Key points}

To our best knowledge, this is the first systematic review to focus on the impact of HBT interventions and HBT feasibility among elderly people living at home in Southeast Asian countries and regions. We identified 6 RCTs through a systematic literature search and found that all six trials were conducted in either China or South Korea; no other trials meeting our search strategy were conducted in the other countries within this area. This implies that HBT intervention has not penetrated well in the area, and HBT development across the area may be still in its infancy. Although the exact reason for this is unclear, one promising background factor is the fact that these two countries are leading 
Table 3 Completion rate, reasons for withdrawal and adverse events in the six eligible randomized controlled trials

\begin{tabular}{|c|c|c|c|c|c|c|}
\hline & Hong et al. [30] & Kwon et al. [31] & Peng et al. [32] & Fang et al. [33] & Chen et al. [34] & Lee et al. [35] \\
\hline $\begin{array}{l}\text { Completion rate } \\
(n / n)\end{array}$ & $\begin{array}{l}\text { Intervention: } 85 \% \\
\quad(11 / 13) \\
\text { Control: } 92 \% \\
(12 / 13)\end{array}$ & $\begin{array}{l}\text { Intervention } 1 \\
\quad \text { (fixed exercise): } \\
59 \%(16 / 27) \\
\text { Intervention } 2 \\
\quad \text { (fixed-interactive } \\
\text { exercise): } 80 \% \\
\text { (24/30) } \\
\text { Control: } 79 \% \\
\text { (22/28) }\end{array}$ & $\begin{array}{l}\text { Intervention: } 86 \% \\
\quad(42 / 49) \\
\text { Control: } 84 \% \\
(41 / 49)\end{array}$ & $\begin{array}{l}\text { Intervention: } 85 \% \\
\quad(34 / 40) \\
\text { Control: } 88 \% \\
(35 / 40)\end{array}$ & $\begin{array}{l}\text { Intervention: } 96 \% \\
\quad(26 / 27) \\
\text { Control: } 89 \% \\
(24 / 27)\end{array}$ & $\begin{array}{l}\text { Intervention: } 76 \% \\
\quad(38 / 50) \\
\text { Control: } 86 \% \\
(43 / 50)\end{array}$ \\
\hline $\begin{array}{l}\text { Reason for with- } \\
\text { drawal }(n)\end{array}$ & $\begin{array}{l}\text { Intervention: } 2 \\
\text { back surgery (1) } \\
\text { post-test no-show } \\
\quad(1) \\
\text { Control: } 1 \\
\text { accidental fall (1) }\end{array}$ & $\begin{array}{l}\text { Intervention } 1 \\
\text { (fixed exercise): } \\
11 \\
\text { withdrawal of IC } \\
\text { (7) } \\
\text { loss to follow-up } \\
\text { (4) } \\
\text { Intervention } 2 \\
\text { (fixed-interactive } \\
\text { exercise): } 6 \\
\text { withdrawal of IC } \\
\text { (4) } \\
\text { loss to follow-up } \\
\text { (2) } \\
\text { Control: } 6 \\
\text { withdrawal of IC } \\
\text { (3) } \\
\text { loss to follow-up } \\
\text { (3) }\end{array}$ & $\begin{array}{l}\text { Intervention: } 7 \\
\text { discontinued inter- } \\
\text { vention (3) } \\
\text { loss to follow-up } \\
\text { (4) } \\
\text { Control: } 8 \\
\text { discontinued inter- } \\
\text { vention (4) } \\
\text { loss to follow-up } \\
\text { (4) }\end{array}$ & $\begin{array}{l}\text { Intervention: } 5 \\
\text { excluded (1) } \\
\text { withdrew (3) } \\
\text { lost contact (1) } \\
\text { Control: } 6 \\
\text { excluded (1) } \\
\text { withdrew (4) } \\
\text { lost contact(1) }\end{array}$ & $\begin{array}{l}\text { Intervention: } 1 \\
\text { refused (1) } \\
\text { Control: } 3 \\
\text { stroke reoccur- } \\
\text { rence (1) } \\
\text { refused (1) } \\
\text { lost contact (1) }\end{array}$ & $\begin{array}{l}\text { Intervention: } 12^{\mathrm{a}} \\
\text { delayed medical } \\
\text { history report (2) } \\
\text { Control: } 7 \\
\text { personal reason (2) }\end{array}$ \\
\hline Adverse events & No description & No description & $\begin{array}{l}\text { No patient had } \\
\text { any significant } \\
\text { complications, } \\
\text { adverse outcomes } \\
\text { or problems } \\
\text { with the use of } \\
\text { QQ and WeChat } \\
\text { software during } \\
\text { the program }\end{array}$ & No description & $\begin{array}{l}\text { No therapy-related } \\
\text { adverse events } \\
\text { were recorded } \\
\text { during the entire } \\
\text { process }\end{array}$ & No description \\
\hline
\end{tabular}

IC informed consent

${ }^{\text {a }}$ Reasons for other drop-outs are not described

countries in internet usability. As of June 2020, China had the largest percentage of internet users in ASIA (854 million people representing $37.1 \%$ of the total Asian population), and South Korea has the highest internet penetration rate of 96.0\% (the average rate across Asian countries and regions is $53.7 \%$ ) [26]. The favorable internet usability environment in these two countries may have boosted HBT intervention and its development.

In the present review, compared with conventional rehabilitation, the HBT interventions showed either equal or better effects on elderly people's physical function with an acceptable rate of intervention completion. Although we could not obtain conclusive evidence due to limited relevant information with wide heterogeneity across the studies, our findings suggest that HBT could be an alternative strategy for the delivery of rehabilitation services for elderly people living at home in the area. We believe the present study provides an accurate systematic overview of the currently available evidence and suggests certain prospects for further research in this field.

\section{Effects}

The primary finding of this study was that HBT is likely to play an active role in improving physical function among elderly people in Southeast Asian countries and regions. This finding is in line with previous systematic reviews that reported positive impacts of remotely monitored cardiac telerehabilitation (CR) [23] and telemedicine [24] among elderly people. Batalik et al. [23] systematically reviewed articles on remote CR programs and reported in their narrative-based analysis that remote $\mathrm{CR}$ programs appear to be 


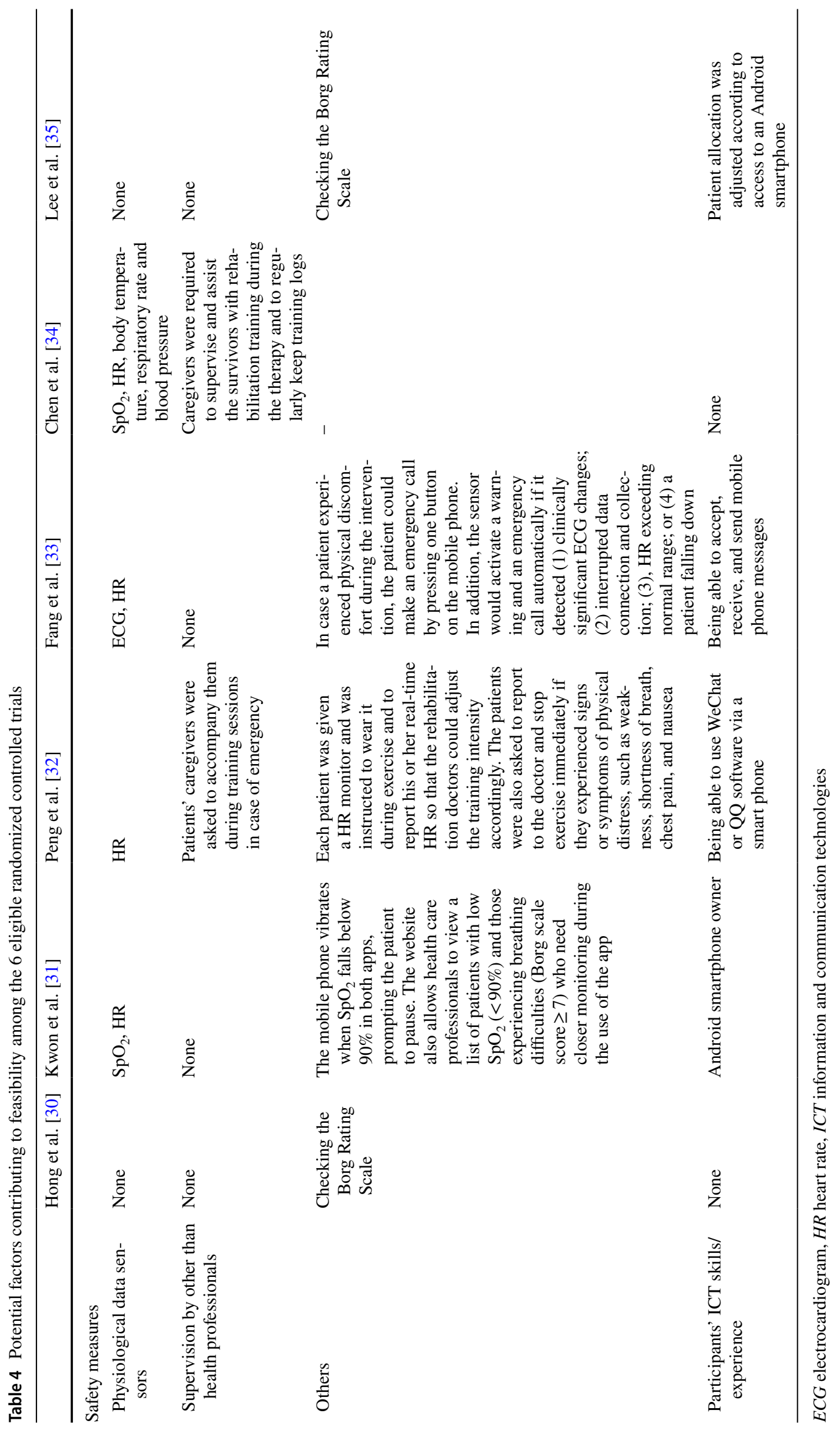


at least as effective in improving cardiovascular risk factors and exercise capacity as traditional center-based programs among patients with an average age of 60 years. Similarly, Batsis et al. [24] conducted a systematic review aiming to reveal the potential of telemedicine (including telerehabilitation) and concluded that telemedicine is a useful modality of health service delivery to older adults. Importantly, however, the findings of these previous systematic review studies were based on RCTs mainly conducted in Western countries and could not necessarily provide specific evidence relevant for Southeast Asian countries and regions. The present systematic review revealed additional evidence that HBT appears to be as effective as other conventional service modalities among Southeast Asian elderly populations, similar to those in Western countries.

Although our review provides relevant evidence that helps to fill a gap in knowledge regarding the effectiveness of HBT among elderly people in Southeast Asia, the methodological quality of the included studies did not allow us to obtain robust evidence as $83 \%(5 / 6)$ of the studies were judged to have "High" risk of bias. Furthermore, the heterogeneity of the participants' conditions, intervention regimens and outcomes did not allow us to conduct a meta-analysis or obtain a definite conclusion. Thus, careful consideration is required when interpreting our findings. The poor methodological quality of and heterogeneity in telehealth-related studies was also reported in previous systematic reviews $[10,18,20$, 21]. Batsis et al. [24] insisted that there is a critical need for high-quality studies investigating the impact of telemedicine interventions in older adults. Performance of further studies taking into account these implications is crucial.

\section{Feasibility}

In our review of the feasibility data across the included studies, the intervention completion rate was available in all studies and was approximately $80 \%$ (range 59-96\%) in the HBT intervention group. This figure appears to be comparable with a previously reported figure for the HBT completion rate among elderly people with mild to moderate disability in Australia (92\%) [37], those with mild cognitive impairment in Italy (65\%) [38], those with acquired brain injury in Israel (62\%) [39] and those suffering from acute myocardial infarction in Italy (100\%) [40]. The systemic review by Jonker et al. [10] also reported the completion rate of perioperative eHealth interventions among elderly people in Western countries, in which, unfortunately, telerehabilitation intervention was not necessary included, to be $54-95 \%$. Although a direct comparison of findings between the present study and these previous studies is difficult due to discrepancies in the intervention regimens, participants' conditions and countries, our findings were consistent with these previous studies. Our findings imply that HBT intervention might be an acceptable rehabilitation strategy for elderly people in Southeastern Asian countries and regions.

In contrast, there was less emphasis on reporting other aspects of feasibility such as the specific reasons for withdrawal from the intervention and adverse events during the intervention. The CONSORT 2010 guideline for reporting of RCTs [41] recommends that researchers describe this information. These descriptions are crucial and informative for clinical practitioners and researchers because this information enables them to make their intervention more userfriendly and safe, and to prevent further drop-outs/accidents in their clinical practice or research project. Crotty et al. [37] described specific reasons for withdrawal from their HBT intervention among elderly people, such as "one person withdrew from the program after finding the technology too stressful" and "one person withdrew because the technology was perceived as being too challenging". These description allow readers to judge the appropriateness of the intervention and can provide an opportunity to simulate it or design a program more feasible for their own clinical practice or research project. No studies in our review provided specific information on withdrawal, and few studies described the occurrence of adverse events during the interventions, indicating that these aspects of the feasibility of HBT interventions may not necessarily be documented well in this field. Further studies documenting this relevant information that can contribute to improving the feasibility of HBT interventions are necessary.

\section{Potential factors contributing to feasibility}

Safety issues, which include medical and technical issues, are pivotal for successful HBT interventions and can be a potential contributing factor to feasibility [17, 28, 42]. Indeed, according to in-depth interview studies investigating elderly patients' experiences with a HBT intervention [43] and a program of computer literacy and education [44], some elderly people are concerned about these issues during the remote telehealth intervention, as indicated by statements such as "some equipment (e.g., dumbbell) might hit themselves on the head" [43] and "it is important that technical assistance is organized if I have problems using the system" [44]. These factors can potentially influence their acceptance of, adherence to and completion of HBT interventions [43, 45].

The present study showed that safety measure during the HBT intervention varied across the included studies. Given the nature of the participants' varied health conditions in the examined studies, observing various kinds of safety measure across the studies is rational, and certain trends were observed.

First, more emphasis on monitoring vital signs was present during the HBT intervention for elderly people with 
cardiovascular and pulmonary diseases. Use of these kinds of real-time monitoring systems during remote interventions has been reported in previous studies focusing on heart disease $[28,46]$ and chronic obstructive pulmonary disease [47]. Piotrowicz [46] argued that supervision using a remote monitoring system during remote interventions for patients with heart failure is a mandatory element for its success.

Second, in a few studies, supervision by other than health professions is required during the HBT intervention. Such supervision during remote intervention was also requested in another previous study to ensure participant safety [48]. Tousignant et al. [48], in their proof-of-concept study of telerehabilitation for older adults, reported that a research assistant was in the home of their participants during their telerehabilitation sessions for safety reasons. Eventually, although no adverse incidents occurred during the delivery of the telerehabilitation services, they insisted that a nonprofessional person (spouse, child, etc.) should be present at home to facilitate and optimize some of the interactions between the clinician and the participants [48]. Moreover, the Australia Physiotherapy Association also recommends supervision by a family member or caregiver during remote interventions as an example of a safety measure to mitigate the risk of falling [49].

To our knowledge, there is no global standard describing medical safety measures during the HBT interventions. As a first principle viewpoint, however, it is rational to believe that the client's safety needs to be comparable to that when service providers are physically present with the client [17, 49]. Thus, safety measures should be tailored to individual participants' conditions and should be varied depending on their conditions during the HBT interventions, as with hospital- or institution-based intervention.

In terms of safety issues relating to technology, we found that eligibility criteria regarding ICTs skills/experience was imposed in two-thirds of the included studies, and only elderly people who appeared to be competent in using ICTs were likely to be enrolled in these trials. From the viewpoint of researchers, imposing these inclusion criteria is understandable to prevent technology-related problems and drop-outs during the intervention and to ensure successful completion of their clinical trials. However, these inclusion criteria may also impact the feasibility of HBT interventions and may hinder clinical practitioners in obtaining true figures on the feasibility of a HBT intervention.

Technology-related issues are a common challenge when planning and implementing a HBT intervention for elderly people because of concerns about usability, adherence and availability of the technology [50]. These concerns can arise from functional decline due to normal aging (e.g., in cognitive function, hearing/vision and gross motor skills), sequelae of their health condition for which HBT intervention is required [51] and their unfamiliarity/apprehension when using the technology [45]. Moreover, gender [52] and socioeconomic variables [50] can influence the usability of digital health technology. To capture true figures regarding the feasibility of HBT interventions, further studies that take these variables into account and examine their influence on patient acceptance of, adherence to and completion of HBT interventions are needed.

\section{Limitations}

There are some limitations to this systematic review. First, some publications may have been missed from our review due to limited use of research databases and language bias. Although our electronic literature search was conducted on PubMed/MEDLINE and PEDro, other research databases such as EMBASE and the Cochran Library were not used due to accessibility issues. Moreover, our review only included articles written in English or Japanese and, thus, did not cover articles written in other languages, especially local languages of the Southeast Asian countries and regions. Given that our focus is Southeast Asian countries and regions, this language bias might seriously impact our results and must be acknowledged when interpreting them. Second, the focus of our review was limited to physical function, as an outcome measure of HBT, and completion rate of the intervention, as an index of the feasibility of HBT. Other outcome measures, such as age-related pathological condition of skeletal muscle (e.g., sarcopenia) [53], activity of daily living and quality of life, and feasibility indexes, such as intervention acceptance, adherence and service satisfaction, should be studied. Furthermore, cost-effectiveness should also be evaluated in further studies. Third, we only included elderly people living in their own home, and thus, our findings cannot be generalized to elderly people living in specific institutions such as a nursing home. Finally, as previously mentioned, we could not conduct a meta-analysis due to heterogeneity across the included studies. HBT interventions need to be conceptualized, coded, classified and grouped in a similar way to ensure their homogeneity and to enable researchers to conduct both direct comparisons between research and meta-analyses.

\section{Conclusion}

In the present review, the HBT intervention showed effects on physical function comparable to those of conventional rehabilitation and an acceptable rate of intervention completion by elderly people living at home in Southeast Asian countries and regions, implying that HBT intervention may have the potential to become an alternative strategy for the delivery of rehabilitation services to these elderly people. These encouraging findings should be supported by further 
uniformly conceptualized studies with high-quality methodology to provide conclusive evidence on the effects and feasibility of HBT.

Supplementary Information The online version contains supplementary material available at https://doi.org/10.1007/s40520-021-01820-3.

Author contributions TS and KPI designed the study, collected and analyzed the data, interpreted the results, and prepared the manuscript. KPI supervised data analysis and manuscript preparation.

Funding No funding was received for conducting this study.

Availability of data and material All data generated or analyzed during this study are included in this published article and it supplementary information files.

Code availability Not applicable.

\section{Declarations}

Conflict of interest The authors have no relevant financial or non-financial interests to disclosure.

Ethics approval Not applicable.

Informed consent For this type of study formal consent is not required.

Consents to participate Not applicable.

Consents to publication Not applicable.

Human and animal rights Not applicable.

\section{References}

1. United Nations, Department of Economic and Social Affairs, Population Division (2020) World Population Ageing 2019 (ST/ ESA/SER.A/444). https://www.un.org/en/development/desa/ population/publications/pdf/ageing/WorldPopulationAgeing2019Report.pdf. Accessed 12 Jan 2021

2. Yarnall AJ, Sayer AA, Clegg A et al (2017) New horizons in multimorbidity in older adults. Age Ageing 46:882-888

3. Rowe JW (2015) Successful aging of societies. Daedalus 144:5-12

4. Christensen K, Doblhammer G, Rau R et al (2009) Ageing populations: the challenges ahead. Lancet 374:1196-1208

5. Cameron I, Crotty M, Currie C et al (2000) Geriatric rehabilitation following fractures in older people: a systematic review. Health Technol Assess 4:1-111

6. Baztán JJ, Suárez-García FM, López-Arrieta J et al (2009) Effectiveness of acute geriatric units on functional decline, living at home, and case fatality among older patients admitted to hospital for acute medical disorders: meta-analysis. BMJ 338:b50

7. World Health Organization: The need to scale up rehabilitation (2017) World Health Organization. https://apps.who.int/iris/handle/10665/331210. Accessed 12 Jan 2021

8. Bright T, Wallace S, Kuper H (2018) A systematic review of access to rehabilitation for people with disabilities in low- and middle-income countries. Int J Environ Res Public Health 15:2165
9. de Toledo P, Jiménez S, del Pozo F et al (2006) Telemedicine experience for chronic care in COPD. IEEE Trans Inf Technol Biomed 10:567-573

10. Jonker LT, Haveman ME, de Bock GH et al (2020) Feasibility of perioperative eHealth interventions for older surgical patients: a systematic review. J Am Med Dir Assoc 21:1844-1851.e2

11. Schwamm LH, Holloway RG, Amarenco P et al (2009) A review of the evidence for the use of telemedicine within stroke systems of care: a scientific statement from the American Heart Association/American Stroke Association. Stroke 40:2616-2634

12. Agostini M, Moja L, Banzi R et al (2015) Telerehabilitation and recovery of motor function: a systematic review and meta-analysis. J Telemed Telecare 21:202-213

13. Steventon A, Bardsley M, Billings J et al (2012) Effect of telehealth on use of secondary care and mortality: findings from the Whole System Demonstrator cluster randomised trial. BMJ 344:e3874

14. Sanders C, Rogers A, Bowen R et al (2012) Exploring barriers to participation and adoption of telehealth and telecare within the Whole System Demonstrator trial: a qualitative study. BMC Health Serv Res 12:220

15. Turolla A, Rossettini G, Viceconti A et al (2020) Musculoskeletal physical therapy during the COVID-19 pandemic: is telerehabilitation the answer? Phys Ther 100:1260-1264

16. O'Neil J, Canadian Physiotherapy Association (2021) TeleRehabilitation in Times of COVID-19. Canadian Physiotherapy Association. https://physiotherapy.ca/times-covid-19. Accessed 12 Jan 2021

17. College of Physical Therapists of British of Columbia (2020) Telerehabilitation guide. https://cptbc.org/wp-content/uploads/ 2020/07/CPTBC_Telerehabilitation-Guide_Final-July-27.pdf. Accessed 12 Jan 2021

18. Pastora-Bernal JM, Martín-Valero R, Barón-López FJ et al (2017) Evidence of benefit of telerehabitation after orthopedic surgery: a systematic review. J Med Internet Res 19:e142

19. Block VA, Pitsch E, Tahir P et al (2016) Remote physical activity monitoring in neurological disease: a systematic review. PLoS One 11:e0154335

20. de Araújo AVL, Neiva JFO, Monteiro CBM et al (2019) Efficacy of virtual reality rehabilitation after spinal cord injury: a systematic review. Biomed Res Int 2019:7106951

21. Appleby E, Gill ST, Hayes LK et al (2019) Effectiveness of telerehabilitation in the management of adults with stroke: a systematic review. PLoS One 14:e0225150

22. Nordio S, Innocenti T, Agostini M et al (2018) The efficacy of telerehabilitation in dysphagic patients: a systematic review. Acta Otorhinolaryngol Ital 38:79-85

23. Batalik L, Filakova K, Batalikova K et al (2020) Remotely monitored telerehabilitation for cardiac patients: a review of the current situation. World J Clin Cases 8:1818-1831

24. Batsis JA, DiMilia PR, Seo LM et al (2019) Effectiveness of ambulatory telemedicine care in older adults: a systematic review. J Am Geriatr Soc 67:1737-1749

25. International Telecommunication Union (2020) Measuring digital development: facts and figures 2020. https://www.itu.int/en/ITUD/Statistics/Pages/facts/default.aspx. Accessed 12 Jan 2021

26. Internet World Stats (2020) World internet usage and population statistics. https://www.internetworldstats.com/stats.htm. Accessed 12 Jan 2021

27. Liberati A, Altman DG, Tetzlaff J et al (2009) The PRISMA statement for reporting systematic reviews and meta-analyses of studies that evaluate health care interventions: explanation and elaboration. J Clin Epidemiol 62:e1-e34

28. Piotrowicz E, Piepoli MF, Jaarsma T et al (2016) Telerehabilitation in heart failure patients: the evidence and the pitfalls. Int $\mathrm{J}$ Cardiol 220:408-413 
29. Higgins JPT, Savović J, Page MJ, Sterne JAC on behalf of the RoB2 Development Group (2019) Revised Cochrane risk-of-bias tool for randomized trials (RoB 2). https://www.riskofbias.info/ welcome/rob-2-0-tool/current-version-of-rob-2. Accessed 12 Jan 2021

30. Hong J, Kim J, Kim SW et al (2017) Effects of home-based teleexercise on sarcopenia among community-dwelling elderly adults: body composition and functional fitness. Exp Gerontol 87:33-39

31. Kwon H, Lee S, Jung EJ et al (2018) An mHealth management platform for patients with chronic obstructive pulmonary disease (efil breath): randomized controlled trial. JMIR Mhealth Uhealth 6:e10502

32. Peng X, Su Y, Hu Z et al (2018) Home-based telehealth exercise training program in Chinese patients with heart failure: a randomized controlled trial. Medicine (Baltimore) 97:e12069

33. Fang J, Huang B, Xu D et al (2019) Innovative application of a home-based and remote sensing cardiac rehabilitation protocol in Chinese patients after percutaneous coronary intervention. Telemed J E Health 25:288-293

34. Chen J, Jin W, Dong WS et al (2017) Effects of home-based telesupervising rehabilitation on physical function for stroke survivors with hemiplegia: a randomized controlled Trial. Am J Phys Med Rehabil 96:152-160

35. Lee BJ, Park YH, Lee JY et al (2019) Smartphone application versus pedometer to promote physical activity in prostate cancer patients. Telemed J E Health 25:1231-1236

36. Jin W, Chen J, Shi F et al (2015) Home-based tele-supervising rehabilitation for brain infarction patients (HTRBIP): study protocol for a randomized controlled trial. Trials 16:61

37. Crotty M, Killington M, van den Berg M et al (2014) Telerehabilitation for older people using off-the-shelf applications: acceptability and feasibility. J Telemed Telecare 20:370-376

38. Mosca IE, Salvadori E, Gerli F et al (2020) Analysis of feasibility, adherence, and appreciation of a newly developed tele-rehabilitation program for people with MCI and VCI. Front Neurol 11:583368

39. Beit Yosef A, Jacobs JM, Shenkar S et al (2019) Activity performance, participation, and quality of life among adults in the chronic stage after acquired brain injury-the feasibility of an occupation-based telerehabilitation intervention. Front Neurol 10:1247

40. Giallauria F, Lucci R, Pilerci F et al (2006) Efficacy of telecardiology in improving the results of cardiac rehabilitation after acute myocardial infarction. Monaldi Arch Chest Dis 66:8-12

41. Schulz KF, Altman DG, Moher D, CONSORT Group (2010) CONSORT 2010 statement: updated guidelines for reporting parallel group randomised trials. BMJ 340:c332
42. Richmond T, Peterson C, Cason J et al (2017) American Telemedicine Association's Principles for Delivering Telerehabilitation Services. Int J Telerehabil 9:63-68

43. Shulver W, Killington M, Morris C et al (2017) "Well, if the kids can do it, I can do it": older rehabilitation patients' experiences of telerehabilitation. Health Expect 20:120-129

44. Cimperman M, Brenčič MM, Trkman P et al (2013) Older adults' perceptions of home telehealth services. Telemed J E Health 19:786-790

45. Nelson MJ, Crossley KM, Bourke MG et al (2017) Telerehabilitation feasibility in total joint replacement. Int J Telerehabil 9:31-38

46. Piotrowicz E (2012) How to do: telerehabilitation in heart failure patients. Cardiol J 19:243-248

47. Zanaboni P, Dinesen B, Hjalmarsen A et al (2016) Long-term integrated telerehabilitation of COPD Patients: a multicentre randomised controlled trial (iTrain). BMC Pulm Med 16:126

48. Tousignant M, Boissy P, Corriveau H et al (2006) In home telerehabilitation for older adults after discharge from an acute hospital or rehabilitation unit: a proof-of-concept study and costs estimation. Disabil Rehabil Assist Technol 1:209-216

49. Australian Physiotherapy Association (2020) Telehealth Guidelines Response to COVID-19 March 2020. https://australian. physio/sites/default/files/APATelehealthGuidelinesCOVID19 0420FA.pdf. Accessed 12 Jan 2021

50. Levine DM, Lipsitz SR, Linder JA (2016) Trends in seniors' use of digital health technology in the United States, 2011-2014. JAMA 316:538-540

51. The American Occupational Therapy Association Advisory Opinion for the Ethics Commission (2017) State Licensure, Professionalism, and the Occupational Therapy Code of Ethics. https:// www.aota.org/ /media/Corporate/Files/Practice/Ethics/Advisory/ State-Licensure-Professionalism-and-Code-of-Ethics-AdvisoryOpinion.pdf. Accessed 12 Jan 2021

52. Koch S, Hägglund M (2009) Health informatics and the delivery of care to older people. Maturitas 63:195-199

53. Kern H, Barberi L, Löfler S et al (2014) Electrical stimulation counteracts muscle decline in seniors. Front Aging Neurosci 6:189

Publisher's Note Springer Nature remains neutral with regard to jurisdictional claims in published maps and institutional affiliations. 